\title{
Stakeholders' perspectives on biobank-based genomic research: systematic review of the literature
}

\author{
Alma Husedzinovic ${ }^{\star, 1,2}$, Dominik Ose ${ }^{3}$, Christoph Schickhardt ${ }^{1}$, Stefan Fröhling ${ }^{2}$ and Eva C Winkler ${ }^{1}$ \\ The success of biobank-based genomic research is widely dependent on people's willingness to donate their tissue. Thus, \\ stakeholders' opinions should be considered in the development of best practice guidelines for research and recruiting \\ participants. We systematically analyzed the empirical literature describing different stakeholders' views towards ethical \\ questions with regard to type of consent, data sharing and return of incidental findings. Patients are more open to one-time \\ general consent than the public. Only a small proportion desires recontact if the research aim changed. A broad consent model \\ would prevent only a small proportion of patients from participating in research. Although professionals are concerned about a \\ risk of reidentification, patients and the public support data sharing and find that the benefit of research outweighs the potential \\ risk of reidentification. However, they desire detailed information about the privacy protection measures. Regarding the return of \\ incidental findings, the public and professionals focus on clinically actionable results, whereas patients are interested in \\ receiving as much information as possible. For professionals, concrete guidelines that help managing the return of incidental \\ findings should be warranted. For this it would be helpful addressing the different categories - actionable, untreatable and \\ inheritable diseases - upfront with patients and public.
}

European Journal of Human Genetics (2015) 23, 1607-1614; doi:10.1038/ejhg.2015.27; published online 4 March 2015

\section{INTRODUCTION}

Technical advances in whole-genome sequencing enable the identification of genomic variations involved in the development of diseases, such as acquired mutations that are causally linked to cancer. ${ }^{1,2}$ Improvements in disease prevention as well as diagnostic and therapeutic strategies may eventually lead to more precise, individually stratified health care. As most diseases are complex, which means that they are caused by multiple genomic factors, research is often based on large numbers of samples representing large numbers of individuals to arrive at statistically significant conclusions. In addition, genetic susceptibility alone is not sufficient to induce a complex disease; the causes also include different lifestyle and environmental factors. Therefore, to study complex diseases the interplay of all of these factors has to be considered. An efficient tool to achieve this is the creation of biobanks. Biobanks are defined as organized collections of human biological specimens comprised of cells, tissues, blood or DNA, which could be linked to clinical data and detailed individual lifestyle. Biobanks vary with respect to types and sources of samples and size, as well as research focus. Biobanks can be disease-specific or population-based, ranging from small collections to large-scale repositories. ${ }^{3-7}$

Linking biological materials to personal data for enabling biobankbased genomic research raises several ethical questions that are currently debated. The focus of the ethical debate is on informed consent as an expression of self-determination of research participants and privacy protection as a part of their personal rights. ${ }^{3,6,8}$ For informed consent to be meaningful, research ethics demands that the participant be informed about the purpose and the content of the project and about its potential risks and benefits. This enables the participant to determine whether to participate or not. ${ }^{3,9-11}$ However, specific consent as recommended by the Declaration of Helsinki ${ }^{11}$ is oftentimes not feasible in biobank-based genomic research: first, as explained above, genomic research improves with larger data sets. Therefore, it is of fundamental importance that research data are shared and can be accessed by as many researchers as possible. Hence, biological specimens and the genomic data can be used for a number of different research purposes in multiple future projects. Not all of them can be specified by the time of consenting. ${ }^{3}$ Second, broad data sharing raises concerns about the protection of the participants' identity and privacy. As the human genome has inherent selfidentifying properties, data protection measures such as deidentification and even anonymization cannot completely protect the privacy of the participant. This means that reidentification of the participants is a remote but potential risk of biobank-based genomic research.,12-15 Third, genomic information is predictive of genomic dispositions to diseases ${ }^{16,17}$ and might be used to discriminate against and stigmatize individuals with certain genomic conditions. ${ }^{8}$ Additionally, genomic research results reveal information that might be of clinical importance to the research participants. These include intended results as well as unintended findings, the so-called 'incidental' findings. ${ }^{16-18}$

In summary, the information about the specific aim of the research, about its risks and benefits, as well as the information about disclosure of incidental findings during the informed consent process, constitute a specific challenge in biobank-based genomic research. $3,9,12,17-20$

The success of biobank-based genomic research is widely dependent on people's willingness to donate their tissue or blood samples and their trust in research and responsible handling of the ethical issues. Therefore, it is necessary that the opinion of patients who donate

\footnotetext{
${ }^{1}$ Programme for Ethics and Patient-Oriented Care in Oncology, National Center for Tumor Diseases (NCT), Heidelberg University Hospital, Heidelberg, Germany; ${ }^{2}$ Department of Translational Oncology, National Center for Tumor Diseases (NCT), German Cancer Research Center (DKFZ), Heidelberg University Hospital, Heidelberg, Germany; ${ }^{3}$ Department of General Practice and Health Services Research, Heidelberg University Hospital, Heidelberg, Germany

*Correspondence: Dr A Husedzinovic, Programme for Ethics and Patient-Oriented Care, National Center for Tumor Diseases (NCT), Heidelberg University Hospital, Im Neuenheimer Feld 460, Heidelberg 69120, Germany. Tel: +49 6221/5635022; Fax: +49 6221/567225; E-mail: Alma.Husedzinovic@nct-heidelberg.de

Received 28 July 2014; revised 19 January 2015; accepted 27 January 2015; published online 4 March 2015
} 
tissue for disease-specific research or of the public involved in population-based studies are considered in the development of best practice guidelines for professionals involved in research and recruiting participants. The aim of this review was to assess the perspectives on ethical aspects of biobank-based genomic research of the relevant stakeholders. These are patients and participants from the general public who donate their tissue for research, as well as professionals involved in research. In particular, we were interested in stakeholders' opinions on the content and process of informed consent, data sharing and privacy protection, and managing of incidental findings. Therefore, we performed a systematic search and analysis of the current empirical literature on this topic.

\section{MATERIALS AND METHODS}

\section{Search strategy}

Our search strategy consisted of two steps. First, a search of the electronic database Web of Science was performed. As we learned from test searches that articles were not uniformly indexed, we adopted a multistep approach, using different combinations of the following keywords: 'informed consent', 'incidental findings', 'data share', 'privacy', 'genome' and 'biobank' (for details on the search strategy, cf. Supplementary Figure S1). Only articles published in English by January 2014 were chosen, of which we selected the 20 most cited and the 20 most recent publications for the extended search in the second step; here we screened the selected papers' reference lists. Subsequently, duplicates were removed and titles and abstracts were independently reviewed for eligibility by two reviewers (AH and EW). ${ }^{21}$

\section{Eligibility criteria}

Articles were considered eligible if stakeholders' perspectives on (1) informed consent requirements in genomic research and/or (2) data sharing and/or privacy protection and/or (3) managing of incidental findings in biobank-based genomic research in general, independent of the type of the biobank, were addressed. Articles addressing these aspects in contexts other than biobankbased genomic research were excluded. Also, articles addressing ethical aspects of biobank-based genomic research with samples and data of minors were excluded from the analysis because the scope of ethical and regulatory questions differs considerably in this group from those of adult participants. Of the remaining studies, full-text articles were analyzed and both reviewers (AH and EW) screened all studies to confirm their relevance to the systematic review. Studies were further classified into articles analyzing informed consent documents and guidelines, empirical studies and theoretical/opinion papers.

\section{Data extraction and content analysis}

Data extraction and content analysis of the empirical studies were conducted independently by two researchers (AH and $\mathrm{DO})$. The extracted data included author, year of publication, the group which was interviewed, method, study setting and sample size and aim of the study (cf. Supplementary Tables S1 and S2). ${ }^{21}$ Subsequently, studies were categorized according to the group of participants, namely patients, public and professionals involved in research. Study findings related to three main topics were analyzed in detail and summarized in the text: (1) informed consent requirements for biobank-based genomic research, (2) opinions on data sharing and privacy protection and (3) views on the management of incidental findings.

\section{RESULTS}

The number of papers identified at each stage of the review process is shown in Figure 1. A total of 837 articles were identified as potentially eligible in the initial search. After the screening of titles and abstracts and the removal of duplicates, 152 articles met the eligibility criteria and were selected for further analysis. Full-text review of all 152 articles identified 40 empirical studies. Fifteen articles analyzing informed consent documents and biobank guidelines and 97 theoretical/opinion papers were excluded from the content analysis, but incorporated in the Discussion section (Figure 1).

\section{Content analysis of articles included}

Among the 40 articles included, 19 were qualitative and 21 were quantitative empirical studies. Data extracted from these studies are shown in Supplementary Tables S1 and S2. Below, we report findings pertaining to each of three main topics: informed consent requirements in biobank-based genomic research, data sharing/privacy protection and return of incidental findings. The main findings are represented in Tables 1, 2 and 3.

Informed consent requirements in biobank-based genomic research Concerning the type of consent, the majority of patients in quantitative studies agreed to a one-time general consent, which allows future research on any subject to ethics approvals. A reconsent for every future research project was regarded as waste of time, effort and money. However, a relevant minority (10-20\%) in these studies wished to be reconsented for each new study ${ }^{22-24}$ to feel more involved and respected and to increase their trust in the institution. ${ }^{23}$ Two studies reported that $10-30 \%$ of patients preferred to have a list of disease research areas so that they could choose which one to support with their biological material. ${ }^{23,24}$ In one study with 1193 patients from five academic health centers in which the patients' attitudes towards the usage of existing samples were assessed, up to $81 \%$ of patients wanted to know if their samples would be used for the research. Their motives included curiosity, confidentiality concerns and patients' right to know the aim of research. ${ }^{25}$ These findings are further supported with additional quantitative studies in which patients whose biological samples were archived during their clinical treatment also requested to be informed about storage and research uses of their samples. ${ }^{26,27}$

In contrast to patients, up to $44 \%$ of respondents from the public requested reconsent for each new study. ${ }^{28-30}$ Furthermore, in one study with the public, $10 \%$ desired a possibility to decline research with their biological specimens in undesirable research fields. ${ }^{30}$ These findings were supported by several other quantitative studies, which reported the public's desire to know the aim of research either as described in informed consent document or as approved by research ethics committee (REC). ${ }^{30-32}$ In one qualitative study with the public, those surveyed expected to be reconsented if the aim of study changed. We do not know, however, from this study how specific the aim was defined. ${ }^{33}$ In the view of the public, additional prerequisites for the participation in biobank-based genomic studies included study approval by the responsible REC and trust in authorities. ${ }^{31,32}$

\section{Data sharing and privacy protection}

Understanding data sharing: benefits and concerns. The majority of the patients and the public had positive attitudes towards biobank-based genomic research and associated data sharing and they were willing to donate their biological samples to research for altruistic reasons. ${ }^{24-27,31,33-38}$ These findings were also supported by the results of various qualitative studies in which patients and the public recognized data sharing in biobank-based research as providing benefits for future generations, science and health. ${ }^{33,36,39-42}$

Regarding concerns and potential risks of biobank-based genomic research and associated data sharing, in several quantitative and qualitative studies, the three groups (patients, the public and professionals) expressed different views ranging from no concerns to raising concerns pertaining to insurance, employment, access to medical care, the disclosure of genomic information about ethnic heritage and stigmatization. $22,24,36,38,40,42-45$ In two qualitative studies, patients assigned higher priority to the benefit of data sharing for science and society than to potential risks. ${ }^{40,43}$ Similar findings were reported 


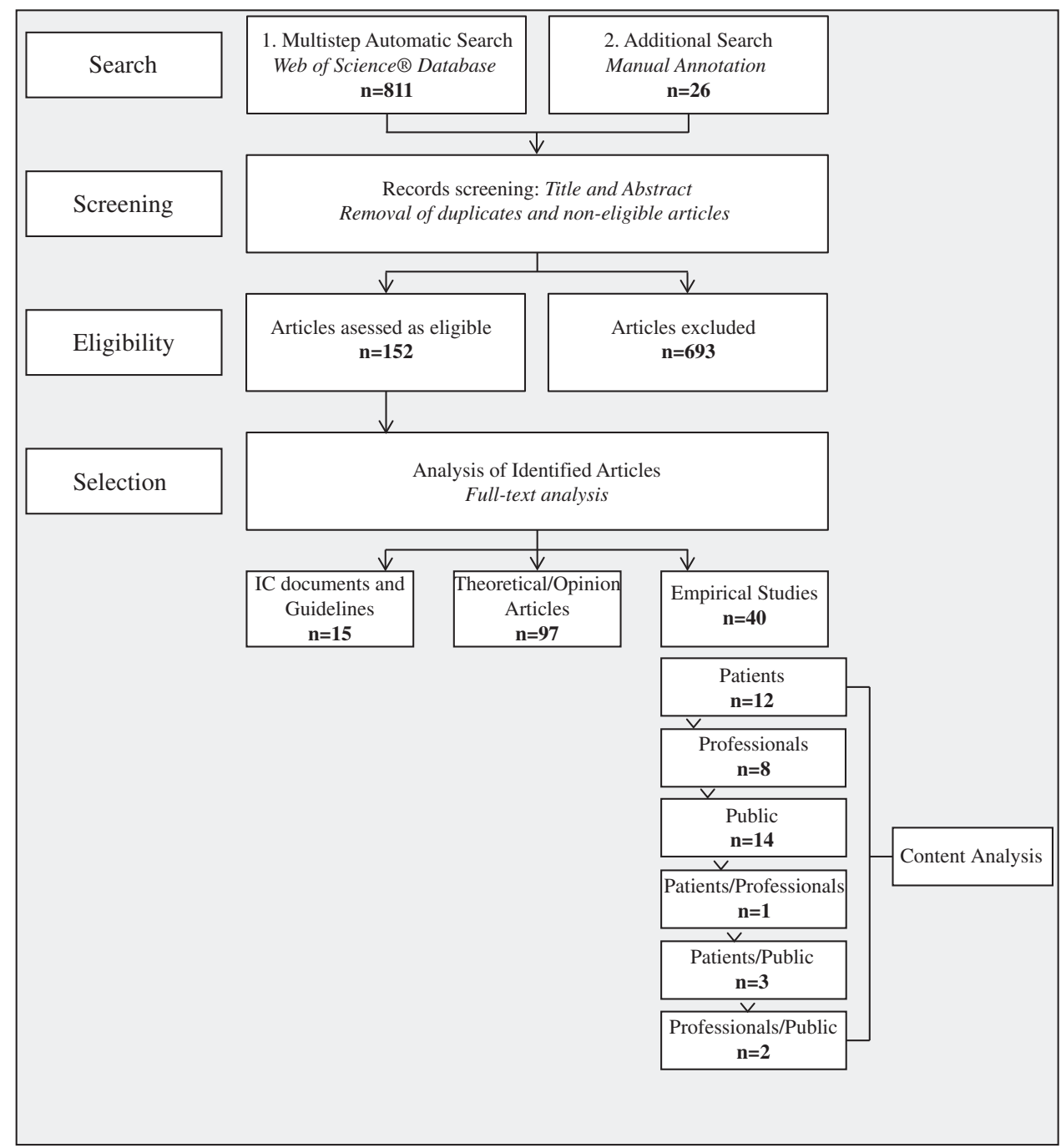

Figure 1 Flow diagram showing the study selection process and classification of identified full-text articles. A total of 837 articles were identified as potentially eligible in the initial search. After the screening of titles and abstracts and the removal of duplicates, 152 articles met the eligibility criteria and were selected for further analysis. The full-text review identified 40 empirical studies, 15 articles analyzing informed consent documents and 97 discussions/ opinion papers. The empirical studies described different stakeholders' perspectives on biobank-based genomic research. Depending on the group that was interviewed, studies were subdivided into the following categories: 'patients' $(n=12)$, 'professionals' $(n=8)$, 'public' $(n=14)$, 'patients/professionals' $(n=1)$, 'patients/public' $(n=3)$ and 'professionals/public' $(n=2)$.

\section{Table 1 Informed consent requirements in biobank-based genomic research: main findings}

Informed consent requirements in biobank-based genomic research

(a) The majority of patients accepted one-time-general consent as appropriate type of consent; reconsent for every future research project was regarded as a waste of time, effort and money ${ }^{22-24}$

(b) Up to $44 \%$ of respondents from the public requested reconsent for each new study ${ }^{28-30}$

(c) Patients and public wanted to know the aim of the of research25-27,30-32

(d) General requirements for participations in research were study approval by REC and trust in authorities 31,32

in quantitative studies with the public. Although concerns about privacy protection in genomic research exist, they do not influence public's willingness to consent and were rated least important. ${ }^{38,46}$ Furthermore, in quantitative studies with patients and the public, respondents expressed a great sense of trust in non-profit or publicly funded research organizations as well as in doctors, hospitals and university-based researchers. However, they had little or no trust in for-profit organizations, insurance companies and the pharmaceutical industry. ${ }^{23,25,31,47}$ The same was reported in several other qualitative studies. ${ }^{37,40,48}$

Preferences for data sharing: data-sharing options. Despite positive attitudes towards data sharing, ${ }^{40-42,49}$ most respondents in quantitative as well as in qualitative studies stressed the importance of being informed about data-sharing practices and privacy protection measures during the informed consent process. ${ }^{30,39-43}$ In several studies, 
Data sharing/privacy protection in biobank-based genomic research

(a) Patients and the public had positive attitudes towards biobank-based genomic research and associated genomic data sharing and were mainly altruistically motivated to participate in research $24-27,29,33-38,42$

(b) Data sharing was recognized as a benefit by patients and the public $31,33,36,40-42,49$

(c) Patients, the public and professionals expressed privacy concerns focused on insurance, employment, 22,24,39,42,43,45 access to medical care and stigmatization $38,40,42-44$

(d) Benefit of data sharing for science and society outweighed potential risks; ${ }^{40,43}$ concerns about privacy do not influence willingness to participate in biobank-based genomic research 38,46

(f) Trust in non-profit or public-funded research organizations ${ }^{23,25,31,37,40,47,48}$

(g) During the informed consent process, it is necessary to have information about data-sharing practices and privacy protection measures and applicable sanctions if the data are misused $30,33,39-43$

Table 3 Return of incidental findings from biobank-based genomic research: main findings

Return of incidental findings from biobank-based genomic research

(a) Patients mostly desired to receive all types of incidental findings $22,23,27$

(b) Although they were interested in receiving incidental findings, general public considered findings about untreatable diseases and results with unknown significance as an unnecessary burden ${ }^{50}$

(c) REC professionals supported return of incidental findings if they were valid, clinically significant, treatable or preventable and if participants wanted to receive them 51

(d) The reasons in favor of return of incidental findings expressed by patients and public were the possibility to make one's life plans, to prevent diseases, empowerment, respect for persons, and also the right to receive this information $43,50,52$

(e) Concerns regarding return of incidental findings identified by patients and public, as well as professionals were diagnostic and therapeutic misunderstanding, uncertainty of results, emotional and psychological harm, and loss of public trust in research institutions $33,50,51,53-56$

(f) The public and professionals, in general, agreed that researchers have the obligation to return incidental findings $47,50,52-54,57$

(g) In the view of the professionals, complexity of data gained from genomic research makes information about the return of incidental findings during the informed consent process difficult 55

(h) Participants should have the possibility to choose whether or not to receive incidental findings ${ }^{56}$

(i) Distinct guideline how to return incidental findings would be helpful, ${ }^{55}$ as well as support structure ${ }^{54}$

important issues that have to be addressed were identified. Patients expected that their genomic data would not be shared without their permission. ${ }^{30,41}$ Information about privacy protection and applicable sanctions if the data were misused were also expected; this was requested by patients as well as by the public..$^{30,33,39}$ Moreover, the public expected additional criteria to be met before data were shared, such as to have consented to the research aim, security measures and trust in the institution storing the data. ${ }^{33}$

\section{Return of incidental findings}

Analyzing the studies on stakeholders' perspectives on return of incidental findings, we observed that several studies used the generic term 'return of results' instead of 'incidental or additional findings'. However, based on characteristics of the results described in these studies, it seems reasonable to assume that authors meant unintended or incidental findings.

Preferences for returning incidental findings. Concerning incidental findings that might indicate a predisposition for diseases, most patients in several quantitative studies wished to be informed. ${ }^{23,27}$ Considering the different types of incidental findings, most of the patients wanted to be informed about curable and incurable diseases, as well as about treatable and untreatable results. ${ }^{22,26}$ The magnitude of a risk to develop a certain disease did not influence the patients' preference. ${ }^{26}$ In contrast, public opinion about results of untreatable diseases and results with unknown significance differed considerably. Some respondents from the qualitative study with public considered this information as an unnecessary burden, whereas others stressed the importance of informing participants about such findings, as over time and with progress in research, information could become more accurate and the related diseases treatable. ${ }^{50}$ Also, REC professionals supported the return of incidental findings if the results were valid, clinically significant, treatable or preventable and if the participants wanted to receive them. ${ }^{51}$

Regarding the reasons in favor of receiving incidental findings, patients and the public had similar perspectives, namely that the return of incidental findings would allow them to make decisions in family planning and take preventive actions to avoid certain diseases. $^{43,52}$ In addition, empowerment, respect for persons, a better public recognition of research and a feeling of control were expressed as arguments for the return of incidental findings. ${ }^{50,52}$

However, several concerns were also emphasized by the public in qualitative studies, such as diagnostic and therapeutic misunderstanding, uncertainty of results and anxiety, which may be caused by the return of incidental findings, or even the inability to pay for a treatment after the diagnosis. Unnecessary additional testing and loss of time were also identified as serious concerns. ${ }^{33,50}$ Professionals shared the concerns about potential emotional and psychological harm to the patients in several quantitative and qualitative studies. ${ }^{51,53-56}$ Some felt that this could in turn lead to a loss of public trust in research institutions. ${ }^{54}$ The most prominent concern of professionals was the complexity of the results, the uncertainty in their interpretation and concerns about the quality of information, including validity and clinical utility of genomic findings. ${ }^{51,54-56}$

The public and professionals, in general, agreed that a researcher has the obligation to disclose incidental findings; ${ }^{47,50,52-54,57}$ however, 
they had different views on which findings should be returned. While the public considered it an obligation to inform about research findings even if the results were not unambiguous ${ }^{57}$, most professionals agreed on the researcher's duty to return 'life-threatening' findings as well as findings on preventable or treatable diseases. ${ }^{53,58}$

Information about the return of incidental findings from genomic research during the informed consent process. Very few qualitative studies with professionals and the public addressed whether returning incidental findings should be discussed with the participant during the informed consent process. Professionals agreed that the large volume of information gained from genomic research as well as the low likelihood to find incidental findings if the analysis is targeted make it difficult to explain possible findings before testing. ${ }^{55}$

Specific recommendations included the explanation of incidental findings as well as the information about the sometimes limited validity and clinical utility of the result. Professionals also thought that participants should be informed about possible benefits and risks, such as emotional, psychological and financial harm that might be induced by the disclosure of incidental findings. They should also have the opportunity to choose whether or not to receive incidental findings. ${ }^{56}$ In a qualitative study, respondents from the public proposed that they should have the opportunity to change their previous decisions regarding receiving results. ${ }^{50}$

How to return incidental findings from biobank-based genomic research. The majority of patients and respondents from the public were of the opinion that the findings should be disclosed to the research participant as well as to his/her physician. ${ }^{23,47}$ In a study with different professionals from multiple research institutions, the majority reported that they had already been involved in the disclosure of incidental findings, but only to physicians and not patients. Almost half of them stated that there was no clear procedure for managing incidental findings and two-thirds agreed that a specific guideline would be helpful. ${ }^{55}$

However, professionals were concerned about the communication of these results and suggested that support structures could be useful. In addition, some practical aspects were considered, such as time and funds for recontacting. ${ }^{54}$ Furthermore, REC professionals explained that they could facilitate the process of return of incidental findings but were not the ones to decide about which findings to return. They suggested that the management of incidental findings required coordinated action of scientific and medical researchers, genomic counselors and medical geneticists. ${ }^{51}$

\section{DISCUSSION}

Biobank-based genomic research generates some ethical challenges, which reflect the tension between the advancement of scientific knowledge and the protection of research participants. Since the success of biobank-based genomic research depends on publics' and patients' trust and their consent to participate in research, $3,5,9,10,12$ it is important to factor stakeholders' views and concerns into the design of best-practice recommendations and governance tools of biobanks storing samples for genomic research. Below, we discuss the empirical findings of stakeholders' perspectives against the background of the ethical debate that was mapped with a focus on the three areas of debate - type of consent, data sharing and privacy protection, and handling of incidental findings.

\section{Type of the consent}

The Declaration of Helsinki on human research demands that all research participants must be informed about the purpose, benefits and risks of research before deciding upon research participation ${ }^{11}$. As specific information about future projects is not available at the time of consent, there is much ethical controversy about the type of informed consent that can permit biobank-based genomic research. ${ }^{3,9,10,12,19,59}$ Some argue that consent is not valid if it does not offer specific information. Therefore, either a narrow or specific type of consent or obtaining new consent from participants for each new project is demanded. ${ }^{60,61}$ Others claim that a broad consent by which participants allow the usage of their biological samples and data in general terms for future studies are still sufficient for participants to make a choice - provided that participants have an opportunity to withdraw, every new study is approved by a REC and personal data are protected..$^{10}$ Empirical studies are not apt to settle an ethical debate, but they can inform the debate. In our analysis, we could show that patients and the public differed in their approval of a broad consent model: although the vast majority of patients (80-90\%) approved broad or one-time general consent, only $\sim 50 \%$ of survey participants representing public opinion did so. Patients were also more considerate about the efforts of recontacting than the public. This is interesting, as it indicates a pattern that we saw in data sharing as well, namely that patients were more supportive of research than the public. This could be because of sampling bias, but it might also reflect that as individuals develop disease and participate in research projects, their views on the research process shift to a more supportive role. The remaining small, but relevant proportion of patients (10-20\%) and $\sim 50 \%$ of the public, however, wished to be recontacted for reconsent if the aim of research changed.

For those participants who require information about the aim of research, it is apparently sufficient to describe it in broad terms (eg, 'cancer research'); thus reconsent would only be necessary if the sample was used in other research fields (eg 'dementia research'). Another way to reconcile seemingly incompatible views would entail offering the option to choose the field of research in which the samples or data can be used. ${ }^{30}$ This information would then need to be stored with the sample, for example, as metadata. Thus, it seems that presenting a broad consent model would prevent only a small proportion of patients to donate their sample to biobanks-based genomic research. If centers want to convince all potential donors (patients and public) to participate, they might want to consider ways to link the information about areas of research that are supported by the donor to the sample. This might be more cost-efficient and less time-consuming than reconsenting patients with specific consent.

\section{Data sharing and privacy protection}

Genomic research carries the risk of reidentification of research participants and thereby may also constitute a threat to their privacy. ${ }^{5,13,15,20}$ In addition, genomic data may include risk-related information about further medical conditions, which may be used in ways that could be harmful to the participant or his/her relatives. ${ }^{3,8,12}$

Our analysis of empirical studies showed that patients as well as the public recognized the possible risk of reidentification and were concerned about a negative impact on their insurance, employment, access to medical care and about stigmatization. They emphasized the importance of being informed about data-sharing practices, measures for privacy protection and applicable sanctions if the data were misused. Interestingly, patients regarded reidentification as a negligible risk. In their evaluation, the benefit of data sharing for science and society outweighed the potential risk and they were generally very 
supportive of data sharing. So far, we have neither informally nor formally reported evidence for data breaches, apart from the proof of principle that reidentification is feasible in some cases with considerable effort. ${ }^{14,62}$ Hence, patients' weighing of scientific benefits over the risk to their privacy seems a well-considered judgment. Unless we experience evidence for serious data breaches, rigid terms of data protection would therefore constitute paternalism over patient preferences and an unjustified obstruction to research. Regarding the regulation of data sharing in biobank-based genomic research, there seems to be a fine line between the need for protection and problematic paternalism.

\section{Return of incidental findings}

Another field of the ethical debate is the handling of incidental findings that might become clinically relevant for research participants. Based on the general ethical principle that the participant has a right to decide whether he or she would want to learn about genetic results (right to know and not to know), the central questions are whether to return these findings at all, and if so, which results to return and how to do so. ${ }^{16-18,20,63,64}$ There is a debate about the criteria that should be decisive for returning findings since the findings differ in their validity, predictive power and clinical significance. The complexity of these characteristics requires expertise for interpreting the implications on health and diseases and makes their communication all the more challenging. ${ }^{12,17,65}$

According to our analysis, the majority of patients and respondents representing the public wanted to receive incidental findings. However, while the respondents from the public recognized the return of findings with unknown significance or untreatable results as an unnecessary burden, patients tended to desire receiving all findings regardless of their characteristics. The difference might be explained by a change in information-seeking behavior when being confronted with a serious illness.

Although professionals were willing to disclose findings, they were concerned about the interpretation and quality of potential results with respect to their validity and clinical utility. They also anticipated possible psychological and emotional harm caused by uninformed communication of disease risks and highlighted the requirement of economic resources for the return of incidental findings. Interestingly, the latter aspect was neither mentioned by patients and respondents from the public nor was it addressed in the studies.

So far, there are different suggestions how to handle the return of incidental findings that range from mandatory testing of a list of genetic diseases and return of the results to no reporting back since we are in a research context. Since we learned from our analysis that primarily patients have an interest to learn about genetic results, a concept of tiered consent that explains different categories of results (eg treatable, untreatable, inheritable with respective penetrance) during the informed consent process would allow patients to specify the type of results they would be interested in or would not want to know. This statement could then guide the counseling if something relevant was found. However, it is ethically questionable whether genetic findings of uncertain significance should be reported back, as these are not 'results' with any clinical meaning, do not contribute to the information status of the patients and still might generate fears and uncertainties. Furthermore, it would be interesting to find out if the preferences towards return of incidental findings changed if the respondents were informed about economic aspects.

For professionals, processes that guarantee the validation of findings out of the research context and guidelines that help interpret their clinical relevance should be warranted. ${ }^{16,20,63}$ If incidental findings gain in importance and frequency, one suggestion would be to install a committee or an interdisciplinary consultation platform for the interpretation of and decision about returning incidental findings, and to set up a multifaceted follow-up support structure for patients, which comprises genomic counseling, psychological support or information about possible treatment. In general, future research needs to underpin the rational that returning (actionable) incidental findings improves the health status of the respective population on the long run.

\section{Limitations}

Our study has some methodological limitations. First, we focused our literature search on the Web of Science database, which allows access to publications across different research disciplines and covers more than 12000 journals worldwide, followed by additional manual annotation of relevant papers. Therefore, our search covers a broad spectrum of publications on this topic, but we cannot exclude that we could identify additional studies if we searched additional databases or screened additional papers' reference lists; however, we chose this approach for practicability reasons and we are of the opinion that with this search we covered the important articles on this topic.

Second, although we provide a comprehensive overview of published studies on stakeholders' perceptions and views on biobankbased genomic research, these studies are prone to sampling biases and might not represent the attitude of the respective countries' population. It is, for example, known that the older population is generally underrepresented in clinical studies. Third, we excluded studies describing attitudes towards the participation of minors in biobank-based genomic research, as this research raises special ethical concerns. And last, the significance of empirical data about participants' preferences needs to be evaluated against other valuable arguments - normative, practical and financial ones. We therefore started discussing these arguments in the Discussion section. However, it is our opinion that practice patterns should not be transformed immediately based simply on empirical studies on patients' and publics' preferences. Still, these preferences are valuable and important information that should inform the ethical debate and, eventually, our policies and governance structures.

\section{CONCLUSION}

To inform further policy work for biobank-based genomic research, we systematically analyzed the existing empirical literature describing different stakeholders' perspectives on the ethically contested questions in the following fields: type of consent, data sharing and privacy protection and managing the return of incidental findings. We found that patients and the public differed considerably in their preferences regarding type of consent and return of results: Patients were more open to one-time general consent than the public, and only a small proportion of patients wanted to be recontacted if the research aim changed. With regard to the return of incidental findings, patients were interested in receiving as much information as possible, whereas the public was more focused on actionable results and more concerned about the potential psychological harm caused by uncertain or untreatable conditions. Professionals shared these concerns. In contrast, while professionals were more concerned about a potential risk of reidentification of participants in genomic research, patients seemed to weigh the need for research higher than their personal privacy.

We identified the following policy implications from our research: presenting a broad consent model would prevent only a small proportion of patients to donate their sample to biobank-based genomic research. If centers want to convince all potential donors (patients and public) to participate, they might want to consider ways 
to annotate the sample with the research area the donor gave his or her consent for and use the sample accordingly. This might be more cost efficient and less time consuming than reconsenting patients with specific consent. To better inform the return of incidental finding process, a concept of tiered consent that explains different categories of results seems to be most appropriate to patients' and publics' preferences. Such categories could include treatable, untreatable and inheritable conditions with information about their penetrance. For professionals, concrete guidelines that help interpret the clinical relevance should be warranted, as well as institutional procedures on how the return of incidental findings should be managed and whose expertise should be involved.

\section{CONFLICT OF INTEREST}

The manuscript has been read and approved for submission by all authors. This manuscript has not been published elsewhere in part or in entirety, and is not under consideration by another journal. The authors declare no conflict of interest.

1 Alexandrov LB, Stratton MR: Mutational signatures: the patterns of somatic mutations hidden in cancer genomes. Curr Opin Genet Dev 2014; 24: 52-60.

2 Garraway LA, Lander ES: Lessons from the cancer genome. Cell 2013; 153 17-37.

3 Cambon-Thomsen A: Science and society - rhe social and ethical issues of postgenomic human biobanks. Nat Rev Genet 2004; 5: 866-873.

4 Budimir D, Polasek O, Marusic A et al: Ethical aspects of human biobanks: a systematic review. Croat Med J 2011; 52: 262-279.

5 Rothstein MA: Expanding the ethical analysis of biobanks. J Law Med Ethics 2005; 33 89-101.

6 Cambon-Thomsen A, Ducournau P, Gourraud PA, Pontille D: Biobanks for genomics and genomics for biobanks. Comp Funct Genom 2003; 4: 628-634.

7 Hodgson SV, Popat S: Polymorphic sequence variants in medicine: a challenge and an opportunity. Clin Med 2003; 3: 260-264.

8 Robertson JA: The $\$ 1000$ genome: ethical and legal issues in whole genome sequencing of individuals. Am J Bioeth 2003; 3: 35-42.

9 Hansson MG: Ethics and biobanks. Br J Cancer 2009; 100: 8-12.

10 Hansson MG, Dillner J, Bartram CR, Carlson JA, Helgesson G: Should donors be allowed to give broad consent to future biobank research? Lancet Oncol 2006; 7: 266-269.

11 Helsinki Do: World Medical Association Declaration of Helsinki: ethical principles for medical research involving human subjects. JAMA 2013; 310: 2191-2194.

12 Greely HAT: The uneasy ethical and legal underpinnings of large-scale genomic biobanks. Annu Rev Genom Hum Genet 2007; 8: 343-364.

13 Heeney C, Hawkins N, de Vries J, Boddington P, Kaye J: Assessing the privacy risks of data sharing in genomics. Public Health Genom 2011; 14: 17-25.

14 Gymrek M, McGuire AL, Golan D, Halperin E, Erlich Y: Identifying personal genomes by surname inference. Science 2013; 339: 321-324.

15 Kaye J: The tension between data sharing and the protection of privacy in genomics research. Annu Rev Genom Hum Genet 2012; 13: 415-431.

16 Wolf SM, Crock BN, Van Ness B et al: Managing incidental findings and research results in genomic research involving biobanks and archived data sets. Genet Med 2012; 14: 361-384.

17 Cho MK: Understanding incidental findings in the context of genetics and genomics. $J$ Law Med Ethics 2008; 36: 208-212.

18 Clayton EW: Incidental findings in genetics research using archived DNA. J Law Med Ethics 2008; 36: 286-291.

19 Henderson GE: Is informed consent broken? Am J Med Sci 2011; 342: 267-272.

20 McGuire AL, Caulfield T, Cho MK: Research ethics and the challenge of whole-genome sequencing. Nat rev Genet 2008; 9: 152-156.

21 Armstrong R, Hall BJ, Doyle J, Waters E: Cochrane Update. 'Scoping the scope' of a cochrane review. J Public Health 2011; 33: 147-150.

22 Helft PR, Champion VL, Eckles R, Johnson CS, Meslin EM: Cancer patients' attitudes toward future research uses of stored human biological materials. J Empir Res Hum Res Ethics 2007; 2: 15-22.

23 Master Z, Claudio JO, Rachul C, Wang JCY, Minden MD, Caulfield T: Cancer patient perceptions on the ethical and legal issues related to biobanking. BMC Med Genom 2013; 6: 8 .

24 Pentz RD, Billot L, Wendler D: Research on stored biological samples: views of African American and White American cancer patients. Am J Med Genet 2006; 140: 733-739.

25 Hull SC, Sharp RR, Botkin JR et al: Patients' views on identifiability of samples and informed consent for genetic research. Am J Bioeth 2008; 8: 62-70.
26 Vermeulen E, Schmidt MK, Aaronson NK, Kuenen M, van Leeuwen FE: Obtaining 'fresh' consent for genetic research with biological samples archived 10 years ago. Eur $J$ Cancer 2009b; 45: 1168-1174.

27 Vermeulen E, Schmidt MK, Aaronson NK et al: Opt-out plus, the patients' choice: preferences of cancer patients concerning information and consent regimen for future research with biological samples archived in the context of treatment. J Clin Pathol 2009a; 62: 275-278.

28 Hoeyer K, Olofsson BO, Mjorndal T, Lynoe N: Informed consent and biobanks: a population-based study of attitudes towards tissue donation for genetic research. Scand J Public Health 2004; 32: 224-229.

29 Kettis-Lindblad A, Ring L, Viberth E, Hansson MG: Perceptions of potential donors in the Swedish public towards information and consent procedures in relation to use of human tissue samples in biobanks: a population-based study. Scand J Public Health 2007: 35: 148-156.

30 Murphy J, Scott J, Kaufman D, Geller G, LeRoy L, Hudson K: Public perspectives on informed consent for biobanking. Am J Public Health 2009; 99: 2128-2134.

31 Kettis-Lindblad A, Ring L, Viberth E, Hansson MG: Genetic research and donation of tissue samples to biobanks. What do potential sample donors in the Swedish general public think? Eur J Public Health 2006; 16: 433-440.

32 Nilstun T, Hermeren G: Human tissue samples and ethics - attitudes of the general public in Sweden to biobank research. Med Health Care Philos 2006; 9: 81-86.

33 Lemke AA, Wolf WA, Hebert-Beirne J, Smith ME: Public and biobank participant attitudes toward genetic research participation and data sharing. Public Health Genom 2010; 13: 368-377.

34 Lee $\mathrm{Cl}$, Bassett LW, Leng $\mathrm{M}$ et al: Patients' willingness to participate in a breast cancer biobank at screening mammogram. Breast Cancer Res Treat 2012; 136: 899-906.

35 Kerath SM, Klein G, Kern M et al: Beliefs and attitudes towards participating in genetic research - a population based cross-sectional study. BMC Public Health 2013; 13: 114.

36 Lemke AA, Halverson C, Ross LF: Biobank participation and returning research results: perspectives from a deliberative engagement in South Side Chicago. Am J Med Genet 2012; 158A: 1029-1037.

37 Wood F, Kowalczuk J, Elwyn G, Mitchell C, Gallacher J: Achieving online consent to participation in large-scale gene-environment studies: a tangible destination. J Med Ethics 2011; 37: 487-492.

38 Kaufman DJ, Murphy-Bollinger J, Scott J, Hudson KL: Public opinion about the importance of privacy in biobank research. Am J Hum Genet 2009; 85: 643-654.

39 Haga SB, O'Daniel J: Public Perspectives Regarding Data-Sharing Practices in Genomics Research. Public Health Genom 2011; 14: 319-324.

40 Trinidad SB, Fullerton SM, Bares JM, Jarvik GP, Larson EB, Burke W: Genomic research and wide data sharing: views of prospective participants. Gen Med 2010; 12: 486-495.

41 McGuire AL, Hamilton JA, Lunstroth R, McCullough LB, Goldman A: DNA data sharing: research participants' perspectives. Gen Med 2008; 10: 46-53.

42 Kaphingst KA, Janoff JM, Harris LN, Emmons KM: Views of female breast cancer patients who donated biologic samples regarding storage and use of samples for genetic research. Clin Genet 2006; 69: 393-398.

43 Tabor HK, Stock J, Brazg T et al: Informed consent for whole genome sequencing: a qualitative analysis of participant expectations and perceptions of risks, benefits, and harms. Am J Med Genet 2012; 158A: 1310-1319.

44 Coors ME, Raymond KM: Substance use disorder genetic research: investigators and participants grapple with the ethical issues. Psychiatr Genet 2009; 19: 83-90.

45 Godard B, Ozdemir V, Fortin M, Egalite N: Ethnocultural community leaders' views and perceptions on biobanks and population specific genomic research: a qualitative research study. Public Understand Sci 2010; 19: 469-485.

46 Pullman D, Etchegary H, Gallagher $\mathrm{K}$ et al: Personal privacy, public benefits, and biobanks: a conjoint analysis of policy priorities and public perceptions. Gen Med 2012; 14: 229-235.

47 Caulfield T, Rachul C, Nelson E: Biobanking, consent, and control: a survey of Albertans on key research ethics issues. Biopreserv Biobank 2012; 10: 433-438.

48 Erwin DO, Moysich K, Kiviniemi MT et al: Community-based partnership to identify keys to biospecimen research participation. J Cancer Educ 2013; 28: 43-51.

49 McGuire AL, Oliver JM, Slashinski MJ et al: To share or not to share: a randomized trial of consent for data sharing in genome research. Gen Med 2011; 13: 948-955.

50 Murphy J, Scott J, Kaufman D, Geller G, LeRoy L, Hudson K: Public expectations for return of results from large-cohort genetic research. Am J Bioeth 2008; 8: 36-43.

51 Dressler LG, Smolek S, Ponsaran R et al: IRB perspectives on the return of individual results from genomic research. Gen Med 2012; 14: 215-222.

52 Bollinger JM, Scott J, Dvoskin R, Kaufman D: Public preferences regarding the return of individual genetic research results: findings from a qualitative focus group study. Gen Med 2012; 14: 451-457.

53 Meulenkamp TM, Gevers SJK, Bovenberg JA, Smets EMA: Researchers' opinions towards the communication of results of biobank research: a survey study. Eur J Hum Genet 2012; 20: 258-262.

54 Meacham MC, Starks H, Burke W, Edwards K: Researcher perspectives on disclosure of incidental findings in genetic research. J Empir Res Hum Res Ethics 2010; 5: 31-41.

55 Downing NR, Williams JK, Daack-Hirsch S, Driessnack M, Simon CM: Genetics specialists' perspectives on disclosure of genomic incidental findings in the clinical setting. Patient Educ Counsel 2013; 90: 133-138.

56 Simon CM, Williams JK, Shinkunas L, Brandt D, Daack-Hirsch S, Driessnack M: Informed consent and genomic incidental findings: IRB chair perspectives. J Empir Res Hum Res Ethics 2011; 6: 53-67. 
57 Meulenkamp TM, Gevers SK, Bovenberg JA, Koppelman GH, van Hylckama Vlieg A, Smets EM: Communication of biobanks' research results: what do (potential) participants want? Am J Med Genet 2010; 152A: 2482-2492.

58 Brandt DS, Shinkunas L, Hillis SL et al: A closer look at the recommended criteria for disclosing genetic results: perspectives of medical genetic specialists, genomic researchers, and institutional review board chairs. J Genet Counsel 2013; 22 : 544-553.

59 Sheehan M: Can broad consent be informed consent? Public Health Ethics 2011; 4 : 226-235.

60 Caulfield T: Biobanks and blanket consent: the proper place of the public good and public perception rationales. Kings Law J 2007; 18: 209-226.
61 Arnason V: Coding and consent: moral challenges of the database project in Iceland Bioethics 2004; 18: 27-49.

62 Erlich Y, Narayanan A: Routes for breaching and protecting genetic privacy. Nat Rev Genet 2014; 15: 409-421.

63 Bredenoord AL, Kroes HY, Cuppen E, Parker M, van Delden JJM: Disclosure of individual genetic data to research participants: the debate reconsidered. Trends Genet 2011; 27: 41-47.

64 Clayton EW, Ross LF: Implications of disclosing individual results of clinical research. JAMA 2006; 295: 37-38.

65 Kaye J: Genetic research on the UK population - do new principles need to be developed? Trends Mol Med 2001; 7: 528-530.

Supplementary Information accompanies this paper on European Journal of Human Genetics website (http://www.nature.com/ejhg) 